\title{
La biotecnología en el impacto ambiental y su aplicación en el Perú
}

\begin{abstract}
Abad Flores Paucarima*
Julio Vitteri Zumarán**

* Magíster en microbiología por la Universidad Nacional Mayor de San Marcos. Posgrado en biotecnia por la Universidad de La Plata, Argentina. Doctorado por la Universidad Nacional Mayor de San Marcos. Profesor de la Facultad de Ciencias Biológicas de la Universidad Nacional Mayor de San Marcos

** Biólogo por la Universidad Nacional Mayor de San Marcos. Posgrado en administración de la educación por la Universidad de Lima. Profesor de la Facultad de Ingeniería Industrial de la Universidad de Lima.
\end{abstract}

Los microorganismos tienen una amplia versatilidad bioquímica; juegan un papel importante en el ambiente debido a que reciclan la materia a través de los ciclos biogeoquímicos. Esta capacidad ha sido explotada en el manejo del impacto ambiental desde hace varios años, gracias a las tecnologías de biorremediación, las cuales se han perfilado en todo el mundo como una alternativa muy atractiva para la limpieza de suelos y ambientes acuíferos contaminados.

La ventaja más importante de la biorremediación es que los contaminantes orgánicos pueden ser realmente transformados y algunos de ellos completamente mineralizados. Comparada con otras tecnologías, la biorremediación es segura y económica, ya que después del tratamiento los suelos pueden ser reutilizados para el crecimiento de plantas. Un gran número de tecnologías de biorremediación y productos microbianos que han sido aplicados y comercializados exitosamente en países desarrollados, han encontrado obstáculos para ser aplicados en el Perú. Buscar estrategias para estimular la actividad biodegradativa de los microorganismos autóctonos es uno de los tantos retos, pero, al mismo tiempo, representa una gran oportunidad para el desarrollo de proyectos de investigación que puedan ser llevados al campo. 


\section{Introducción}

Durante las dos últimas décadas la calidad de las aguas subterráneas y marinas del litoral costero, en los países industrializados y en vías de desarrollo, se ha deteriorado. Esta degradación está íntimamente relacionada con el crecimiento industrial y con prácticas equivocadas para la disposición final de materiales de desecho de todo tipo.

Los derrames incontrolados de combustibles y aceites en talleres de mecánica, estaciones de servicio y plantas industriales, así como la descarga de aguas residuales industriales en canales o alcantarillas a cielo abierto, también han influido de manera importante en la contaminación del suelo y de las aguas subterráneas con un deterioro significativo de los ecosistemas naturales.

En todo el mundo se ha desarrollado una gran diversidad de tecnologías para la limpieza de suelos y acuíferos contaminados. De acuerdo con su base de funcionamiento se podrían clasificar en: fisicoquímicas, térmicas y biológicas. Dentro de las tecnologías biológicas, la biorremediación ha surgido en los últimos años como una alternativa muy atractiva, debido a que los contaminantes son realmente transformados y algunos completamente mineralizados. En este trabajo se presenta un análisis de los retos y las oportunidades de las tecnologías de biorremediación de suelos y acuíferos en nuestro país.

El Perú enfrenta en la actualidad problemas ambientales muy serios, en un contexto económico que no le permite aplicar en forma generalizada las soluciones de control de contaminación ambiental que son utilizadas en países desarrollados. Sin embargo, existe una gran preocupación e interés por los efectos nocivos que una gran diversidad de compuestos orgánicos tóxicos producen en los recursos hídricos, los suelos y la atmósfera.

$\mathrm{El}$ efecto nocivo de estos compuestos depende de diversos factores, entre los que se incluyen: las propiedades físicas y químicas de los contaminantes, la concentración y las combinaciones de los mismos, la frecuencia y la duración de las emisiones, así como las características del medio en el cual son descargados.

El constante desarrollo tecnológico e industrial, así como el crecimiento excesivo de las ciudades que se observa en el presente, conlleva el incremento de la generación de residuos sólidos tanto de origen doméstico como industrial. La mayor parte 
de los residuos industriales son tóxicos peligrosos que difícilmente son reciclables y por ende provocan problemas serios en su manejo y disposición. No obstante, existen otros desechos, generados principalmente por industrias de alimentos, que podrían ser revalorizados. Sin embargo, en informaciones recientes se ha demostrado que la generación de residuos industriales en las grandes ciudades del Perú se calcula en aproximadamente 40.000 ton/día, de las cuales aproximadamente 1.400 toneladas se clasifican como desechos peligrosos.

Entre los compuestos químicos considerados como altamente tóxicos se encuentran las siguientes categorías: pesticidas, compuestos alifáticos halogenados, compuestos aromáticos (clorados), bifenilos policlorados, nitroaromáticos, ésteres de ftalato, azo-colorantes, nitrosaminas, etc. (Sedue, 1991). Estos compuestos que han sido identificados como contaminantes de aguas residuales, suelo y aire son generados principalmente por las industrias petroquímica, de síntesis orgánica, de textiles, papelera, pinturas, farmacéutica, metalúrgica, producción de explosivos, entre otras. La contaminación generada por estas industrias se ha estimado en más de $20 \%$ del total de las descargas de aguas residuales en la ciudad de Lima, con una importante cantidad de compuestos orgánicos peligrosos y recalcitrantes.

Es conveniente destacar que en la generación de residuos peligrosos se incluyen además compuestos orgánicos volátiles altamente tóxicos que representan un problema importante para el medio ambiente y para la salud pública. Entre las fuentes de emisiones no naturales hacia la atmósfera se pueden distinguir dos tipos: las móviles, como autos, aviones, etc., y las fijas, como las generadas en las industrias, los hogares, etc. Los contaminantes atmosféricos descargados pueden, a su vez, clasificarse en partículas, gases y vapores. Los gases y vapores se encuentran disueltos en la fase gaseosa y pueden ser inorgánicos u orgánicos. Incluyen gases de combustión completa ( $\mathrm{SO}_{2} \mathrm{y}$ óxidos de nitrógeno: $\left.\mathrm{N}\right)$ e incompleta, como el monóxido de carbono y los hidrocarburos. El ozono y otros compuestos orgánicos volátiles (hidrocarburos) son generados por otros procesos no asociados a la combustión.

Como consecuencia de los altos índices de contaminación generados por estos compuestos, las prácticas asociadas con su manejo y disposición han traído consigo diversos efectos de naturaleza socioeconómica y ecológica. Con respecto a la contami- 
nación del agua, la reducción de la disponibilidad de ésta ha provocado una mayor explotación de los mantos acuíferos y el transporte de fuentes lejanas incrementando el costo de suministro. Además, el empleo de aguas residuales contaminadas eleva considerablemente los costos del tratamiento para adecuar este recurso a los usos doméstico e industrial. En lo referente a la contaminación del aire se requiere implementar nuevas tecnologías en las que se incluye la modificación al proceso de producción e instalación de equipo para reducir y/o controlar contaminantes específicos, lo que implica tiempo y un costo adicional muy importante.

\section{Ventajas tecnológicas de la biorremediación}

El suelo y el subsuelo constituyen un recurso natural no renovable que desempeña diversas funciones, entre las que destacan su papel como medio filtrante durante la carga de acuíferos y como capa de protección de éstos; también están integrados al escenario donde ocurren los ciclos biogeoquímicos, hidrológicos y de la cadena alimenticia. Además, el suelo es el espacio donde se desarrollan las actividades agrícolas y ganaderas, las que constituyen la base de la alimentación; y donde también se cultivan áreas verdes que son fuente de regeneración de oxígeno. Como funciones no menos importantes, suelo y subsuelo constituyen el hábitat natural de la biodiversidad y el albergue de la reserva cultural, sin olvidar la función más conocida como la base física para la construcción de edificaciones.

Resulta obvia la necesidad de recuperar un ecosistema contaminado para devolverlo a sus funciones, ya que si un ecosistema se pierde o se destruye, no es imposible hablar de sustentabilidad. Desde este punto de vista, la biorremediación se ubica como una tecnología limpia en la que los contaminantes realmente pueden ser transformados y algunos completamente mineralizados. Además, debido a que se recomienda la utilización de microorganismos cuyo hábitat natural es el suelo, éstos mueren cuando se agotan los nutrientes.

La biorremediación es versátil porque puede adaptarse a las necesidades de cada sitio. Así, puede aplicarse bioestimulación si únicamente se requiere la adición de nutrientes, bioincremento cuando se necesita aumentar la proporción de la flora microbiana degradadora del contaminante, o bien, bioventeo cuando se hace necesario el suministro de oxígeno del aire. 
Además, la biorremediación puede realizarse fuera del sitio cuando la contaminación es superficial, pero necesariamente in situ cuando los contaminantes han alcanzado la zona saturada.

\section{Ventajas económicas de la biorremediación}

Una característica importante de la biorremediación es su bajo costo en relación con otras tecnologías de tratamiento. De acuerdo con Alper (1993), la biorremediación es por lo menos seis veces más económica con respecto a la incineración, y tres veces menor que el confinamiento. Este bajo costo se debe a: un menor gasto de energía; las necesidades de nutrientes son bajas; y que se opera bajo condiciones ambientales. Sin embargo, cabe señalar que la comparación de costos no puede generalizarse, es aplicable sólo para cada caso.

\section{Ventajas ambientales de la biorremediación}

Como se mencionó anteriormente, la biorremediación se ubica como una tecnología limpia debido a que los contaminantes pueden ser realmente transformados en compuestos deseablemente inocuos al ambiente, y algunos de ellos pueden ser completamente mineralizados. Además, los microorganismos mueren cuando se agotan los contaminantes que están siendo empleados como substrato.

En el caso de aguas subterráneas, la tecnología más utilizada es la llamada bombeo-tratamiento, recarga que consiste en extraer el agua subterránea, tratarla en la superficie y posteriormente devolverla al acuífero. A pesar de ser muy empleada existen algunos aspectos que determinan el éxito de su aplicación, por ejemplo: los contaminantes pueden estar fuertemente absorbidos en el material geológico, o bien estar presentes en zonas de baja permeabilidad, lo que ocasiona limitaciones en la transferencia de masa. En otros casos, se hace más difícil poder alcanzar los niveles de limpieza establecidos, debido a que las bajas concentraciones de contaminantes que los microorganismos utilizan como substrato no son suficientes para soportar su actividad microbiana, por lo que éstos empiezan a morir. Además de que se hace muy costoso el tratamiento mismo, por el gasto de energía que implica el bombeo. 


\section{Retos tecnológicos de la biorremediación}

A escala de demostración se ha visto que la biorremediación tiene muy poco éxito cuando existen metales pesados en concentraciones tales que inhiban la actividad microbiana, o bien, cuando las condiciones microambientales sean muy extremas. Debido a que cada microorganismo tiene sus propias características, la tolerancia que presentan a cada situación es muy particular. Por ejemplo, cuando las concentraciones de los contaminantes son muy altas, se observan fenómenos de inhibición.

La biorremediación se hace muy difícil cuando el material geológico es netamente arcilloso, porque su baja permeabilidad limita la transferencia de masa en el sistema. Lo anterior es determinante cuando la contaminación está en el nivel freático y el tratamiento es in situ. Para los suelos superficiales este problema puede superarse si se agrega arena, o bien, residuos agroindustriales que aumentan la permeabilidad y favorecen la transferencia de masa.

Otro aspecto que es necesario resaltar es la complejidad química de la contaminación en diversos sitios, además de que, en algunos casos, los contaminantes tienen ya un avanzado grado de intemperismo, situación que dificulta cualquier tipo de tratamiento.

Para poder participar en proyectos de biorremediación es necesario involucrarse completamente con el problema, tener acceso al sitio contaminado y realizar una profunda investigación sobre los antecedentes del sitio y de las causas que llevaron al problema de contaminación. Esto permite establecer las bases para la completa caracterización del lugar y plantear una estrategia de investigación que conduzca a la solución de un problema real, a través de un desarrollo tecnológico integral para cada caso particular.

En biorremediación no es suficiente trabajar con casos ideales en el laboratorio, es indispensable mantener las condiciones que imperan en el lugar para que el estudio sea representativo del alcance que puede tener en el campo. En este sentido, conviene considerar los siguientes aspectos:

- Los microorganismos autóctonos encargados de la degradación de contaminantes son consorcios microbianos con características propias dependiendo de las condiciones microambientales del lugar donde se encuentran. 
- Preferentemente se debe estimular o enriquecer la población microbiana autóctona, en lugar de aplicar microorganismos exógenos que muy probablemente mueran por la competencia que se establece en el medio natural.

- Los microorganismos generalmente están adheridos a las partículas de suelo y reciben de éste un aporte de microelementos que permiten su sobrevivencia e incluso su actividad metabólica, por lo que se les debe cultivar en las condiciones más parecidas a su hábitat natural.

- Si se decide por el aislamiento de microorganismos y su propagación masiva, éstos no necesariamente se adaptarán al nuevo microambiente donde se depositen.

- De los contaminantes presentes en el sitio, una gran cantidad de ellos serán utilizados por los microorganismos como substratos, pero otra parte podrían quedar sin ser transformados.

- Las fuentes de nitrógeno y fosfatos, preferentemente, deben agregarse en concentraciones limitadas para permitir únicamente la actividad degradativa, que no necesariamente va ligada con el incremento de la población microbiana.

- En biorremediación se debe tener cuidado durante la selección de los compuestos químicos que se adicionen como nutrientes o cosubstratos, ya que éstos pueden crear efectos adversos en el ambiente.

- Se deben adoptar las condiciones microambientales del sitio, aunque no correspondan a las óptimas para el crecimiento y desarrollo microbiano.

- Tener un sitio contaminado es por sí mismo un riesgo, someterlo a una biorremediación implica otro riesgo adicional, por tanto, la idea de aplicar microorganismos manipulados genéticamente (MMG) en el ambiente debe separarlos hasta que se comprendan claramente los mecanismos de transportes y destino que rigen en un medio natural, y se conozcan los riesgos adicionales que implica su uso.

Lo importante en biorremediación es entender que el biorreactor debe ser "construido" en el sitio de tratamiento. Si se trata de una biocelda estará parcialmente confinada, pero cuando se trata de un acuífero el biorreactor no tendrá paredes, tapa ni fondo. Tampoco se podrá trabajar en condiciones asépticas, 
de manera que el concepto tradicional de un biorrector cambia notablemente. Cuando los microorganismos han logrado adaptarse a las condiciones del biorreactor, la biodegradación de los contaminantes ocurrirá en un tiempo que generalmente es largo si se compara con otras técnicas de remediación.

La definición de los parámetros de seguimiento durante el proceso de biorremediación de campo, es otro reto a enfrentar. En lo que respecta a los contaminantes, el enfoque tradicional es seguir la reducción de los compuestos químicos más tóxicos, pero su reducción no necesariamente corresponde a la limpieza del lugar. Un ejemplo clásico se tiene cuando un sitio contaminado con gasolinas va a ser biorremediado, los compuestos químicos indicadores son los monoaromáticos volátiles: benceno, tolueno, etilbenceno y xilenos (BTEX). El aspecto que sufre de inmediato es que la cuantificación de estos compuestos debe hacerse cuidadosamente para evitar resultados falsos, pero también debe hacerse un seguimiento de los hidrocarburos de mayor peso molecular para saber si están siendo degradados. Éstos son dos conceptos diferentes, el primero se refiere a la reducción de la toxina y el segundo a la limpieza del sitio.

\section{Oportunidades de aplicación de la tecnología de la biorremediación en el Perú}

A pesar de que no se cuenta con un inventario de sitios contaminados que sean del dominio público y que sirva de base para la estimación del mercado real de la biorremediación, éste existe. De hecho, esa fue la razón por la que un gran número de compañías extranjeras llegaron al Perú hace algún tiempo, con la finalidad de vender sus tecnologías, sin embargo, los resultados fueron poco exitosos. Las principales causas fueron: bajas eficiencias de reducción de contaminantes, un mayor deterioro ambiental por la adición de químicos y altos costos de operación. Un factor común para muchas de estas compañías fue la aplicación de productos microbianos vendidos como "polvos mágicos" capaces de destruir todo tipo de contaminantes, además de los "aditivos" que son productos químicos patentados de formulación desconocida, los que contienen nutrientes y algunos de ellos tensoactivos, los cuales no siempre son biodegradables y cuando son agregados al suelo ayudan a la dispersión de los contaminantes, más que a su degradación (Saval, 1995). 
Un aspecto indispensable que debe considerarse en cualquier tipo de tecnologías es que para lograr el éxito en su aplicación es necesario realizar estudios para su adaptación e innovación, que puede llevar incluso a nuevos desarrollos. En el caso de las tecnologías de biorremediación debe tomarse en cuenta que las características de cada suelo son diferentes y que no es una regla general que los microorganismos se adapten fácilmente a cualquier hábitat, aquí conviene mencionar que en el caso del Perú, los suelos tienen características físicas, químicas y biológicas muy particulares que los hace diferentes de suelos de otras regiones del planeta.

Para aquellos proyectos de biorremediación a escala real, su costo y duración están en función de los niveles de limpieza requeridos, y éstos dependen de cada caso particular. Obviamente las principales figuras que aparecen en estos proyectos con fines comerciales son la industria contaminadora, la empresa de servicios ambientales y las autoridades respectivas. Actualmente, para el sector académico se han abierto las puertas de nuevas áreas de investigación que podrán generar tecnologías adhoc con un verdadero soporte científico, este esquema permitirá remover en el corto plazo la relación universidad-industria, y crear una importante vinculación con las autoridades ambientales.

Finalmente, cabe señalar que a pesar de la importancia de los problemas ambientales y de la necesidad de soluciones adecuadas a nuestro medio, no existen carreras a nivel licenciatura que permitan la formación de profesionales con una visión muy dirigida a la prevención y control de la contaminación ambiental, mucho menos en lo que se refiere a suelos y aguas subterráneas. Se requiere un enfoque multidisciplinario con una importante integración de áreas como la biotecnología, hidrogeología, geociencia, geotecnia, fisicoquímica y química, entre otras, sin dejar de lado lo referente a legislación.

\section{Consideraciones finales}

La necesidad de enfrentar los difíciles problemas ambientales de los ecosistemas naturales del litoral peruano, basado en principios de sustentabilidad, conforma un marco de referencia en el cual la biorremediación puede contribuir en forma importante a la limpieza de suelos y acuíferos. Para el sector académico se abre la oportunidad de estudiar problemas de contami- 
nación específicos, con miras a desarrollar propuestas de solución aplicables al caso del Perú, en el corto y mediano plazos.

\section{Propuestas de aplicación de la tecnología de biorremediación}

Las propuestas de solución a la problemática actual de contaminación implican la implementación de nuevas estrategias basadas en innovaciones y aplicaciones biotecnológicas que permiten eliminar, reducir o reciclar los compuestos orgánicos tóxicos, con la finalidad de transformarlos a formas compatibles con el ecosistema y que puedan ser asimiladas en los ciclos naturales. A este respecto, se tiene conocimiento de que la flora natural presente en aguas y suelos contaminados es capaz de degradar ciertos compuestos orgánicos, aunque lamentablemente esta posibilidad es moderamente significativa, debido a su alta concentración y toxicidad. Considerando esta capacidad de la microflora natural, se han realizado estudios enfocados a la selección de microorganismos capaces de metabolizar rápidamente estos compuestos como fuente de carbono y de energía.

En las investigaciones realizadas, se han utilizado bacterias en procesos anaerobios y aerobios para la degradación de compuestos aromáticos organoclorados, sin embargo, otros compuestos químicos (compuestos altamente clorados, colorantes, solventes, etc.) son resistentes al ataque bacteriano. La degradación de estas sustancias por hongos ha sido estudiada por diferentes grupos de trabajo (Field et al., 1993; Yadad, 1993). En estudios recientes con Phanerochaete chrysosporium, han demostrado que este hongo no solamente es capaz de degradar la lignina y compuestos similares, sino que también tiene la capacidad para degradar una diversidad importante de compuestos orgánicos, tóxicos y recalcitrantes, tales como clorofenoles, dioxinas clorados, bifenilos policlorados, nitroarométicos, compuestos fenólicos, azo-colorantes, etc. (Field et al., 1993, Paszczynski et al., 1991, Reineke y Knasckmuss, 1988).

La capacidad de estos microorganismos para degradar estas sustancias depende esencialmente de la disponibilidad de un sistema recalcitrante específico (ligninolisis). En estas condiciones la mayor parte de los compuestos orgánicos recalcitrantes pueden ser oxidados y mineralizados por basidiomicetos (Field et al., 1993). La biodegradación ha sido asociada con los diferentes grupos de enzimas que participan en la ligninolisis (peroxidasas y fenoloxidasas). En estas condiciones la degradación 
fue observada durante el metabolismo secundario, inducida por una limitación de la fuente de nitrógeno o de carbono (Chen et al., 1991). En otros estudios realizados sobre la degradación de compuestos aromáticos (compuestos de tipo fenólico) se ha reportado la utilización de fenoloxidadas (tirosinasa y lacasa) producidas por Agaricus bisporus (Shuttlerworth y Bollag, 1986; Wood, 1980). En trabajos recientes se ha confirmado que estas enzimas extracelulares producidas por basidiomicetos son eficaces en el tratamiento de desechos contaminados con compuestos orgánicos recalcitrantes. De hecho, una gran parte de los estudios realizados confirman que los basidiomicetos son excelentes candidatos para la biorremediación de suelos y aguas (Hammael, 1989).

\section{Propuesta de trabajo de investigación y desarrollo biotecnológico de biorremediación de acuíferos contaminados con hidrocarburos residuales y compuestos clorados}

\section{Estudios de caracterización fisicoquímica del efluente}

Para una exitosa aplicación de las tecnologías de biorremediación se tienen que efectuar preliminarmente estudios cuidadosos de caracterización de los efluentes industriales considerando tres aspectos: hidrogeológico, químico y microbiológico.

\section{Caracterización hidrogeológica}

Mediante la aplicación de equipos piezométricos, se podrá conocer la conductividad hidráulica con el fin de determinar la distribución de los contaminantes dentro de una zona contaminada. La toma de puntos de muestreo de zonas cercanas no saturadas, servirá para conocer la estratigrafía y permeabilidad del subsuelo, las mismas que servirán para determinar los coeficientes de absorción. Esta información permitirá obtener un mapa tridimensional de la dispersión de los contaminantes, el cual se deberá actualizar constantemente con un registro piezométrico y químico para confirmar que la reducción del foco de contaminación realmente está ocurriendo por efectos de las medidas de biorremediación. 


\section{Caracterización química}

A través de determinaciones analíticas de Demanda Bioquímica de Oxígeno (DBO); Demanda Química de Oxígeno (DQO); concentración de metales pesados tóxicos: cromo, cadmio, arsénico, plomo, zinc, níquel, molibdeno, etc.; nitratos, cianuros, sulfuros, cloruros, hidrocarburos aromáticos policíclicos; compuestos organo-clorados, etc.; y compuestos orgánicos e inorgánicos que pueden servir como aceptores de electrones para la respiración microbiana por vía anóxica, se podrá determinar la posibilidad de aplicación de la biorremediación.

\section{Caracterización microbiológica}

Para evaluar la posibilidad de aplicar una tecnología de biorremediación es indispensable realizar una caracterización de la biocenosis microbiana (tipos de población microbiana). Esto permite identificar el tipo de metabolismo que sigue la población microbiana, el tipo de aceptor de electrones preferido por la población para efectuar la degradación del contaminante de interés.

En este aspecto, se pondrá especial énfasis en caracterizar las poblaciones de bacterias (pseudomonadales, vibrionaceas, acinetobacter, corynebacterium y actinomycetales), levaduras y hongos del grupo de los phanerochaetes nativos del lugar y que tengan potencial de oxidación de hidrocarburos y compuestos organoclorados.

Es importante señalar que desde el punto de vista práctico, no es suficiente demostrar que hay biodegradación del contaminante en condiciones de laboratorio. Se debe considerar que al desarrollar una tecnología de biorremediación se deberán cubrir tres aspectos fundamentales:

- Se debe contar con una información bien documentada de la pérdida del contaminante in situ, lo cual requiere efectuar un buen seguimiento de los contaminantes en muestras preferentemente inalteradas.

- Realizar ensayos en el laboratorio que demuestren la capacidad de los microorganismos para transformar los contaminantes bajo condiciones que imperan en el sitio contaminado, esto se logra a través de pruebas de biodegradabilidad. 
- Obtener información que demuestre que la biodegradación puede ocurrir realmente en condiciones de campo.

Sólo cuando se cubran estos tres aspectos se podrá pensar en la biorremediación como una alternativa para la limpieza de un sitio contaminado. Una vez que se obtiene esta información, será necesario encontrar las condiciones más apropiadas (optimización) para lograr la máxima degradación del contaminante en el menor tiempo posible y a los costos más bajos.

Es importante señalar que las tecnologías de biorremediación que existen actualmente en el mercado, fueron desarrolladas para sitios específicos, lo cual significa que para poder aplicarlas en otro lugar tendrán que realizarse estudios previos que permitan conocer las características hidrogeológicas, químicas y microbiológicas del lugar donde se pretende aplicar la biorremediación, a fin de hacer las modificaciones o innovaciones necesarias a través de la investigación y llevadas a cabo en laboratorio, antes de proceder a su aplicación a escala de demostración en campo.

\section{Bibliografía}

Chen H., G., C.G. Dosoretz y H.E. Grethelein

"Ligninase Production by Inmobilized Cultures of Phanerochaete Chrysosporium Grown Under Nitrogen-Sufficient Conditions". Enzime Microb. Technol. 13, London: Elsevier Science, 1991, pp. 404-407.

Field, J.M. et al.

"Screening for Ligninolytic Fungi Applicable to the Biodegradation of Xenobiotics". Trends in Biotechnol 11. Cambridge: Elsevier Trends Journal, 1993, pp. 4449.

Fuller, M.E, D.Y. Mu y K.M. Scow

"Biodegradation of Trichloroethylene and Toluene by Indigenous Microbial Populations in Vadose Sediments”. Microb. Ecology 29. London: Elsevier Science, 1995, pp. 311-325. 
Hammael, K. E.

"Organopollutant Degradation by Ligninolytic Fungi". Enzyme, and Microbiol. Technol 11. London: Elsevier Science, 1989, pp. 776-777.

Morales, V. et al.

"Biotechnical Process for the Treatment of H2S and CS2 from a Waste Gas from a Cellophane Plant". Fifht International IGT Symposium on Gas, Oil and Environmental Biotechnology. Chicago: Revash, September 1992.

Paszczynski, A. et al.

"New Approach to Improve the Degradation of Recalcitrant azo Dyes by Streptomyces spp. And Phanerochaete Chrysosporium". Enzyme Microbiol. Technol. 13. London: Elsevier Science, 1991, pp. 378384.

Reineke, W. y H.J. Knasckmuss

"Microbial Degradation of Haloaromatics". Ann. Rev. Microbiol. 42, 1988, pp. 236-287.

Saval, S.

"Remediación y restauración". Pemex: Ambiente y energía. Los retos del futuro. México DF: UNAMPetróleos Mexicanos, 1995, pp. 151-189.

Shuttlerworth, K.L. y J.M. Bollag

"Soluble and Immobilized Laccase as Catalyst for Transformation of Substituted Phenols". Enzyme Microbiol. Technol. 8. London: Elsevier Science, 1989, pp. 171-177.

Solliero, J.L. y R. Castañon

"Environmental Biotechnologies in Mexico: Potencial and Constraints for Development and Difussion". Biotechnology for Sustainable Development. Knoxville: University of Tennessee, 1996.

Sedue

Subsecretaría Ecológica. Norma Técnica Ecológica. Aguas residuales. Dirección General de Planeamiento y Control de la Contaminación Ambiental. NTE-CCA031/91, 1991. 
Wood, D.A.

"Production, Purification and Properties of Extracellular Laccase of Agaricus Bisporus". J. Gen. Microbiol. 117. 1980, pp. 327-338.

Yadad, J.S. y C.A. Reddy

"Degradation of Benzene, Toluene, Ethylbenzene and Xylenes (BTEX) by the Lignin-Degradading Basidiomycete Phanerochaete Chrysosporium". Appl. Environ. Microbiol. 59 (3), 1993, pp. 756-762. 